\title{
Dispositional Greed Scale: evidências de sua estrutura interna e parâmetros dos itens
}

\author{
Leogildo Alves Freires \\ Gleidson Diego Lopes Loureto ${ }^{2}$ \\ Maria Gabriela Costa Ribeiro ${ }^{2}$ \\ Layrtthon Carlos de Oliveira Santos ${ }^{2}$ \\ Valdiney Veloso Gouveia ${ }^{2}$ \\ ${ }^{1}$ Universidade Federal de Alagoas, Maceió, AL \\ ${ }^{2}$ Universidade Federal da Paraíba, João Pessoa, PB
}

\begin{abstract}
Resumo
Este estudo objetivou conhecer as evidências da estrutura interna da Dispositional Greed Scale (DGS) e os parâmetros de seus itens. Contou-se com a participação de 338 pessoas com média de idade de 26,5 anos $(D P=8,18)$, que responderam a DGS e perguntas demográficas. Os resultados indicaram uma solução unifatorial da DGS $(\Omega=0,75)$. Análises via Teoria de Resposta ao Item demonstraram que os itens da DGS discriminam adequadamente os participantes e exigem baixa a moderada quantidade de traço latente para serem endossados. Ademais, detectou-se funcionamento diferencial, quanto à variável sexo, em apenas um item da DGS que apresentou impacto reduzido nas curvas características do teste total. Conclui-se que a DGS reúne evidências que corroboram sua qualidade psicométrica, podendo ser utilizada em estudos futuros que objetivem mensurar a ganância no contexto brasileiro.

Palavras-chave: ganância; adaptação; validade; teoria de resposta ao item
\end{abstract}

Dispositional Greed Scale: evidences of its internal structure and item parameters

\begin{abstract}
This study aimed to investigate the evidences of internal structure of the Dispositional Greed Scale (DGS) and to check the parameters of its items. Participants included 338 individuals with a mean age of 26.5 years old $(\mathrm{SD}=8.18)$, who answered the DGS and demographic questions. Results supported the one-factorial solution of the DGS $(\Omega=0.75)$, and the Item Response Theory analysis demonstrated that the items of the DGS discriminate participants properly and require low to moderate levels of latent trait to be endorsed. Furthermore, only one item displayed differential item functioning by sex, with low impact on item characteristic curves at overall test. In conclusion, the DGS shows evidences of its psychometric adequacy for use in future studies that aim to measure greed in Brazil.

Keywords: greed; adaptation; validity; item response theory
\end{abstract}

Dispositional Greed Scale: evidencias de su estructura interna y parámetros de los ítems

\section{Resumen}

El objetivo de este estudio fue conocer evidencias de la estructura interna de la Dispositional Greed Scale (DGS) y los parámetros de sus ítems. Los participantes fueron 338 personas con edad promedio de 26,5 años (DE $=8,18)$, que respondieron la DGS y preguntas demográficas. Los resultados indicaron una solución unifactorial de la DGS $(\Omega=0,75)$. Los análisis por medio de la Teoría de Respuesta al Item demostraron que los ítems de la DGS discriminan adecuadamente los participantes,y exigen una cantidad baja a moderada de rasgo latente para ser respaldados. Además, se ha identificado el funcionamiento diferencial con relación a la variable sexo en un único ítem de la DGS, que presentó un impacto reducido en las curvas características del test total. Se concluye que la DGS reúne evidencias que confirman su calidad psicométrica, pudiendo ser utilizada en estudios futuros que tengan como objetivo medir la codicia en el contexto brasileño.

Palabras clave: codicia; adaptación; validez; teoría de respuesta al ítem

A ganância é tema de estudo explorado extensamente ao longo da história e nas mais diversas áreas do conhecimento (e.g., filosofia, ética da economia e psicologia; Haynes, Josefy, \& Hitt, 2015). Entretanto, as discussões a respeito têm sido eminentemente teóricas, havendo poucas evidências empíricas sobre suas causas e consequências (Seuntjens, Van de Ven, Zeelenberg, \& van der Schors, 2016). A propósito, apenas a partir de 2010 houve um aumento substancial nos estudos empíricos sobre a ganância (Seuntjens et al., 2016).
No que tange aos aspectos conceituais, o Dicionário Michaelis (Weiszflög, 2015) indica que a ganância é um "Desejo ou ambição desmedida de ganho ou lucro, ambição, avidez, cupidez". Entretanto, esta definição oculta aspectos importantes do construto. Especificamente, definições de ganância oriundas de áreas como filosofia, psicologia, sociologia e economia convergem em um ponto: a insaciabilidade (Krekel \& Pandelaere, 2015). Portanto, o termo se refere a um desejo insaciável por bens e recursos, não apenas por dinheiro 
(Seuntjens, Zeelenberg, Breugelmans, \& Ven, 2014). Nesta direção, um indivíduo ganancioso busca sempre mais, não se restringindo a bens materiais, incluindo entre suas metas o reconhecimento, o poder e o status, não se satisfazendo com o que possui (Seuntjens, Zeelenberg, Van de Ven, \& Breugelmans, 2015).

A ganância é entendida como uma disposição, podendo repercutir na prática de uma série de comportamentos imorais e antiéticos (Marcus \& Zeighler-Hill, 2015), tendo papel, inclusive, para a deflagração de crises econômicas mundiais (Reavis, 2012). De igual modo, a disposição para a ganância é importante para a explicação da propensão a fazer investimentos de risco (Mussel \& Hewig, 2016) e compras por impulso (Seuntjens et al., 2015). Seuntjens e colaboradores verificaram ainda que a ganância se relaciona negativamente com aspectos mais estáveis da personalidade (e.g., autoestima, amabilidade, conscienciosidade, abertura e estabilidade emocional). Tais evidências indicam certa estabilidade da ganância. Ademais, quanto às diferenças entre sexo, estudos prévios (e.g., Krekel \& Pandelaere, 2015; Seuntjens et al., 2015) indicam que a ganância é uma tendência mais proeminente em homens, uma vez que as mulheres são mais orientadas socialmente (altruístas) e os homens possuem uma orientação mais individual (egoísta) (Eckel \& Grossman, 1998).

Apesar de ser um aspecto psicológico sombrio, capturando motivações egoístas (Crocker, Canevello, \& Brown, 2017), a ganância é inerente à natureza humana, de modo que em alguma medida as pessoas possuem este traço latente (Seuntjens et al., 2014). Apesar da relevância de se estudar a ganância, vide que esta pode explicar uma série de comportamentos prejudiciais a milhares de pessoas (e.g., corrupção, delitos, crimes comuns), a literatura sobre o tema ainda é escassa, sobretudo em razão da carência de instrumentos que avaliem esse construto de forma psicometricamente adequada (Mussel \& Hewig, 2016).

A título ilustrativo, no que tange ao contexto nacional (buscas realizadas nas bases de dados IndexPsi, PePSIC, SciELO e LILACS), não foi encontrado qualquer estudo que tenha abordado o construto ganância. Por outro lado, já é possível contar com duas medidas desenvolvidas em estudos recentes no contexto internacional para mensurar este construto (busca realizada na base de dados PsycInfo, utilizando os descritores "greed", "validity" e "scale"), elaboradas por Krekel e Pandelaere (2015) e Seuntjens et al. (2015). Tendo em vista o panorama supracitado, o presente estudo teve como objetivo adaptar ao contexto brasileiro a Dispositional
Greed Scale (DGS; Seuntjens et al., 2015), conhecendo evidências de sua estrutura interna e dos parâmetros de seus itens, neste caso via Teoria de Resposta ao Item (TRI). A escolha deste instrumento se deu em razão de sua adequação teórica e psicométrica, tendo seus itens sido elaborados a partir de estudo prévio que considerou a análise de protótipos do conceito de ganância. Descreve-se a seguir o processo de elaboração da DGS.

\section{Elaboração da Dispositional Greed Scale}

A DGS foi desenvolvida por Seuntjens et al. (2015). Entretanto, antes de testá-la empiricamente, Seuntjens et al. (2014) realizaram cinco estudos com o propósito de conhecer como as pessoas definem a ganância, buscando identificar, sistematicamente, os elementos centrais deste construto. No caso, as pessoas foram orientadas a listar aspectos que pensavam se relacionar com a ganância (Estudo 1), e logo indicando os elementos centrais e periféricos para a sua descrição (Estudo 2). Destas etapas resultaram 46 aspectos relativos à ganância, 23 centrais (e.g., comportamento imoral e egoísmo) e 23 periféricos (e.g., status e vaidade). Em estudos experimentais, os aspectos centrais foram lembrados e reconhecidos de forma mais eficaz do que os periféricos (Estudo 3), além de serem mais rapidamente identificados (Estudo 4). Por fim, no quinto estudo os participantes foram solicitados a indicar eventos nos quais sentiram ganância. Após a descrição da situação, avaliaram em que medida cada um dos 46 aspectos descritores do construto estava presente na situação, sendo que os centrais foram mais presentes que os periféricos. Em síntese, Seuntjens et al. (2014) indicaram que os elementos centrais da ganância foram, por exemplo, o desejo de sempre obter mais, associado à insatisfação de nunca ter o suficiente, abrangendo o anseio por ganhos tanto materiais quanto não materiais.

Considerando a lista proposta por Seuntjens et al. (2014), Seuntjens et al. (2015) elaboraram uma medida de 20 itens para avaliação da ganância, testando-a em quatro estudos. No primeiro, os autores realizaram uma Análise dos Componentes Principais (ACP), encontrando uma solução trifatorial. Ao analisar semanticamente os itens da matriz padrão, estes autores observaram que o primeiro componente, contando com oito itens, reunia indicadores referentes aos elementos centrais da ganância, sendo excluído um item com a menor saturação e que tinha pontuação inversa aos demais, resultando na versão com sete itens. Este novo conjunto de itens foi submetido a uma nova ACP, confirmando-se a solução unifatorial $(\alpha=0,82)$. Nos 
estudos subsequentes, a estrutura unifatorial da versão de sete itens foi replicada, tendo coeficientes alfa de Cronbach aceitáveis, além de estabilidade temporal, atestando a sua adequação. Ainda, tais autores verificaram a validade convergente e discriminante da DGS, observando, por exemplo, correlações positivas com inveja e impulsividade, e negativas com satisfação com a vida e empatia.

Em resumo, conclui-se que a DGS apresenta propriedades psicométricas satisfatórias no seu contexto de origem, tratando-se de uma medida parcimoniosa, composta por sete itens, justificando seu emprego em situações de pesquisa. Diante do exposto e considerando o objetivo do presente estudo, decidiu-se apresentar a adaptação da DGS (Seuntjens et al., 2015) focando em dois eixos: (1) evidências de validade com base na estrutura interna e precisão (estrutura fatorial e consistência interna) e (2) comprovação dos parâmetros (discriminação e dificuldade) e funcionamento diferencial de seus itens. Neste caso, considera-se o sexo dos participantes, um fator potencial de viés em razão de homens e mulheres diferirem em suas pontuações neste construto, conforme indicado previamente.

\section{Método}

Tradução e adaptação da DGS para o contexto brasileiro

Os processos de tradução e adaptação da DGS foram realizados conforme as diretrizes da International
Test Commission (ITC, 2010) recentemente padronizadas em língua portuguesa (Borsa, Damásio \& Bandeira, 2012). Inicialmente, considerando o idioma original da DGS, dois tradutores independentes procederam à tradução dos itens da medida do inglês para o português. Posteriormente, realizou-se a tradução reversa (back translation) do português para o inglês, por um terceiro tradutor independente. Em seguida, comparando-se as versões original e retrotraduzida do instrumento, concluiu-se que as mesmas eram semanticamente equivalentes. Por fim, realizou-se sua validação semântica junto à população-alvo, contando com a participação de oito pessoas. Como desfecho dessa fase, chegou-se à versão final desta escala (Tabela 1).

\section{Participantes}

Contou-se com uma amostra não probabilística de 338 participantes brasileiros da população geral, equiparados quanto ao sexo (169 homens e 169 mulheres), tendo idades variando de 18 a 58 anos $(M=26,5 ; D P$ $=8,10)$, a maioria se declarando heterossexual $(83,7 \%)$, solteira $(73,4 \%)$ e protestante $(32,5 \%)$. Quanto à escolaridade, a seguinte distribuição foi observada: estudantes universitários (49,7\%), ensino superior completo $(33,1 \%)$ e pós-graduação $(17,2 \%)$.

\section{Instrumentos}

Os participantes responderam a um questionário que, além de perguntas sociodemográficas (idade, sexo,

Tabela 1.

Estrutura fatorial da Dispositional Greed Scale (DGS)

\begin{tabular}{clcc}
\hline Itens & \multicolumn{1}{c}{ Descrição do Conteúdo } & Fator I & $\mathrm{h}^{2}$ \\
\hline 1. & O lema da minha vida é: “quanto mais, melhor”. & $0,63(0,55-0,74)$ & 0,40 \\
2. & Quero sempre mais que antes. & $0,76(0,71-, 084)$ & 0,59 \\
3. & Assim que adquiro algo, começo logo a pensar sobre a próxima coisa que desejo. & $0,59(0,44-0,70)$ & 0,35 \\
4. & Não importa o que já tenho, nunca estou completamente satisfeito. & $0,52(0,36-0,64)$ & 0,27 \\
5. & Não me imagino tendo muitas coisas. (R) & $0,54(0,34-0,68)$ & 0,29 \\
6. & Nunca se pode ter dinheiro demais. (R)* & $0,19(0,16-0,37)$ & 0,03 \\
7. & Me considero um pouco ganancioso(a). & $0,39(0,30-0,53)$ & 0,15 \\
\hline Número de itens & 7 & \\
Valor próprio & 2,73 \\
Variância explicada & $40 \%$ \\
$\Omega$ de McDonald & 0,75 \\
\hline
\end{tabular}

Nota. * Item não incluído no cálculo do ômega de McDonald; $(\mathrm{R})=$ itens invertidos; $h^{2}=$ comunalidades. Entre parênteses, intervalos de confiança $(95 \%)$ para as cargas fatoriais, via método bias-corrected and accelerated (bcA) bootstrap.

Psico-USF, Bragança Paulista, v. 24, n. 3, p. 489-500, jul./set. 2019 
orientação sexual, estado civil e religião), apresentava a Dispositional Greed Scale (DGS). Originalmente desenvolvida por Seuntjens et al. (2015), esta escala se compõe de sete itens que descrevem diferenças individuais frente à ganância (e.g., Meu lema de vida é: quanto mais melhor, Quero sempre mais que antes, etc.). Os participantes foram demandados a indicar o seu grau de concordância ou discordância com cada um dos itens, utilizando escala de resposta de cinco pontos, variando de 1 (Discordo totalmente) a 5 (Concordo totalmente).

\section{Procedimento}

A coleta dos dados foi realizada online. As pessoas foram contatadas por meio de redes sociais (e.g., Facebook, Instagram), sendo solicitadas a responder voluntariamente instrumentos autoaplicáveis. Respeitaram-se as prerrogativas dispostas nas resoluções 510/16 do Conselho Nacional de Saúde no que tange à regulamentação de pesquisas com seres humanos, tendo o participante declarado seu consentimento por meio de assinatura de Termo de Consentimento Livre e Esclarecido. A presente pesquisa foi aprovada pelo Comitê de Ética em Pesquisas com Seres Humanos da Universidade Federal de Roraima (Parecer n. 1.440.625). Em média, as pessoas levaram 15 minutos para concluir sua participação no estudo.

\section{Análise dos dados}

Para conhecer as evidências de validade com base na estrutura interna e precisão da DGS, empregou-se Análise Fatorial Exploratória (AFE), considerando o estimador Unweighted Least Squares (ULS), tendo como entrada a matriz de correlações policóricas, em razão da natureza da medida, isto é, escala Likert composta de categorias ordenadas (Holgado-Tello, Chacón-Moscoso, Barbero-García, \& Vila-Abad, 2010). Para a determinação da quantidade de fatores a serem extraídos da DGS, aplicou-se o método Hull, que objetiva encontrar uma estimativa ideal que melhor corresponda a relação entre o ajuste do modelo e o número de parâmetros; este procedimento foi testado através do indicador Comparative Fit Index (CFI $\geq 0,90$; Lorenzo-Seva, Timmerman, \& Kiers, 2011). Por fim, quanto à consistência interna, utilizou-se o índice de ômega de McDonald. Tais análises foram executadas com o Factor (Lorenzo-Seva \& Ferrando, 2013) em sua versão 10.8.01 (2018).

No que tange às análises da TRI, considerou-se o R 3.3.2 (R Development Core Team, 2015), utilizando o pacote estatístico mirt (Chalmers, 2012) para estimar os parâmetros individuais dos itens da DGS (discriminação, dificuldade e curva de informação da DGS), a partir do Modelo de Resposta Graduada (Samejima, 1969), uma vez que se trata de uma escala de resposta politômica. Ademais, investigou-se o DIF dos itens da DGS quanto ao sexo dos participantes, uma vez que tal procedimento é importante para estimar fatores que podem enviesar a interpretação dos resultados de um dado teste (Walker, 2011). No caso, empregou-se o método híbrido de Regressão Logística, que se baseia nos escores estimados via TRI, consistindo em um procedimento mais adequado por não utilizar a simples soma dos escores de um dado teste, como na Teoria Clássica dos Testes (Pasquali, 2007). Basicamente, esta análise identifica itens cuja probabilidade de acerto/ endosso difere entre distintos grupos de uma população, considerando pessoas com o mesmo nível de habilidade na variável de interesse (Walker, 2011).

Os procedimentos para a detecção de DIF foram realizados com o pacote lordif (Choi, Gibbons \& Crane, 2016), adotando-se o método de purificação de Crane et al. (2006), que consiste em cálculos sucessivos dos níveis de traço latente para a equiparação dos thetas dos grupos, de modo a culminar em dois grupos de itens: itens que não apresentam DIF e itens que apresentam DIF. Dessa forma, os itens que supostamente têm um funcionamento diferencial não contribuirão para a pontuação da variável latente (theta). Em suma, este processo possibilita avaliar o impacto dos itens com suposto DIF nas curvas características do teste.

Nesta ocasião, três métodos foram estipulados para detecção do DIF dos itens. O primeiro, o teste de razão de verossimilhança (Swaminathan \& Rogers, 1990), examina três modelos hierárquicos em que a diferença significativa entre os modelos 1 e 2 aponta para a existência de um DIF uniforme, havendo um DIF não uniforme quando os modelos 3 e 2 se diferenciam. Quando somente os modelos 1 e 3 são diferentes, não é possível indicar se um dado item apresenta um DIF uniforme ou não uniforme. O segundo critério consiste no tamanho do efeito entre os modelos a partir do Pseudo- $\mathrm{R}^{2}$, considerando valores menores que 0,035 como um DIF insignificante, valores entre 0,035 e 0,070 como moderados e maiores que 0,070 como grandes (Jodoin \& Gierl, 2001). Finalmente, aplicou-se o critério da diferença entre o parâmetro $\beta_{1}$ dos modelos 1 e 2 em que o $\Delta \beta_{1}$ menores que 0,1 (10\%) (Crane, van Belle \& Larson, 2004) ou 0,05 (5\%) (Crane, Hart, Gibbons, \& Cook, 2006) apontam para a inexistência de DIF.

Psico-USF, Bragança Paulista, v. 24, n. 3, p. 489-500, jul./set. 2019 


\section{Resultados}

Evidências de Validade: Estrutura Interna e Precisão da DGS

Inicialmente, os resultados suportaram o emprego da AFE ordinal ULS, atestando a possibilidade da fatorabilidade da matriz de dados $[K M O=0,70$ e Teste de esfericidade de Bartlett, $\left.\chi^{2}(21)=365,70, p<0,001\right]$. Quanto ao número de fatores a serem retidos do instrumento (método Hull), os dados suportaram uma solução unidimensional com um índice de ajuste adequado para a referida estrutura fatorial $(\mathrm{CFI}=0,93)$.

Em seguida, a partir da inspeção da matriz de dados, observou-se que todos os itens apresentaram cargas fatoriais adequadas $(\lambda \geq 0,30)$, excetuando o item 6 (Nunca se pode ter dinheiro demais), cuja carga foi 0,19. Desta forma, em razão de um critério empírico, o referido item não foi considerado em análises subsequentes. Assim, os seis itens da presente solução fatorial exibiram saturações maiores ou iguais a $|0,39|$, variando de 0,39 [(Item 7. Me considero um pouco ganancioso(a)] a 0,76 (Item 2. Quero sempre mais que antes). Ressalta-se que nenhum dos seis itens restantes apresentou carga fatorial abaixo de 0,30 no limite inferior do intervalo de confiança (Tabela 1). Em síntese, a estrutura fatorial da DGS no contexto brasileiro, composta por seis itens, mostrou-se unifatorial, apresentando valor próprio de 2,73, que foi responsável por explicar $40 \%$ da variância total, com a média de saturações dos itens de 0,57 (DP $=0,12$ ) e coeficiente de consistência interna (ômega de $M c$ Donald, $\Omega$ ) de 0,75 . A seguir, as análises buscam conhecer os parâmetros dos itens da DGS via TRI.

Uma vez assegurada a unidimensionalidade da DGS, prosseguiu-se com a investigação dos parâmetros de seus itens, tendo em conta a TRI. Primeiramente, empregando-se o Modelo de Resposta Gradual de Samejima (1969), estimaram-se seus índices de discriminação (parâmetro a) e de dificuldade (parâmetro b), observando, ainda, a curva de informação do teste. Em um segundo momento, procurou-se conhecer o DIF dos itens da DGS a partir do sexo dos participantes. Tais resultados são descritos a seguir.

\section{Parâmetros dos Itens da DGS: Discriminação e Dificuldade}

Observou-se que os itens da DGS, em conjunto, apresentaram discriminação alta $(M=1,33 ; D P=0,65)$, sendo o menos discriminativo o item 7 ( $a=0,73)$, cuja discriminação foi moderada, e o mais discriminativo o item $2(a=2,50)$, tendo discriminação muito alta. Com relação à dificuldade $\left(\mathrm{b}_{1}-\mathrm{b}_{4}\right)$, ao avaliar os limiares de respostas dos itens, verificou-se a existência de itens que exigiram baixa a moderada quantidade de traço latente $(M=0,11 ; D P=0,63)$, variando entre $-0,97$ (item 5$)$ a 0,90 (item 4). Em síntese, os itens 2, 3 e 5 apresentaram os menores índices de dificuldade, ao passo que os itens 1,4 e 7 exigiram maiores níveis de traço latente para serem endossados. Tais resultados são mostrados na Tabela 2.

No que tange à Curva de Informação do Teste (Figura 1), esta demonstra que a maior quantidade de informação capturada se encontra, aproximadamente, no intervalo de $-4,10$ a 3,90, mostrando-se um instrumento amplamente informativo. Isso significa que a DGS é mais adequada para mensurar a ganância em pessoas que possuem um theta $(\theta)$ no intervalo mencionado. Uma vez conhecidos os parâmetros dos itens

Tabela 2.

Parâmetros dos itens da DGS (Modelo de Resposta Gradual)

\begin{tabular}{cccccc}
\hline Itens & $\mathrm{a}$ & $\mathrm{b}_{1}$ & $\mathrm{~b}_{2}$ & $\mathrm{~b}_{3}$ & $\mathrm{~b}_{4}$ \\
\hline Item 1 & 1,70 & $-1,60$ & 0,04 & 0,95 & 2,61 \\
Item 2 & 2,50 & $-1,70$ & $-0,45$ & 0,11 & 1,88 \\
Item 3 & 1,18 & $-2,22$ & $-0,41$ & 0,26 & 2,69 \\
Item 4 & 0,99 & $-1,91$ & 0,49 & 1,20 & 3,83 \\
Item 5 & 0,92 & $-4,42$ & $-1,48$ & $-0,57$ & 2,56 \\
Item 7 & 0,73 & $-3,50$ & $-0,78$ & 0,29 & 4,83 \\
\hline
\end{tabular}

Nota. $a=$ parâmetro de discriminação; $\mathrm{b}_{1-4}=$ parâmetro de dificuldade

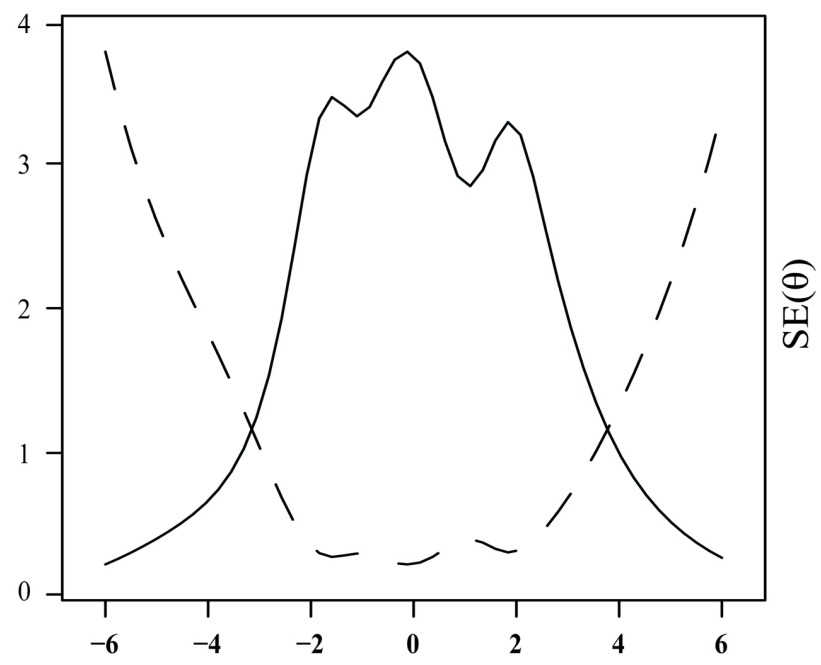

$(\theta)$

Figura 1. Curva de informação do teste da DGS 
desta escala, checa-se a seguir a comprovação de DIF de seus itens.

\section{Funcionamento Diferencial dos Itens: Sexo dos Participantes}

Inicialmente, definiu-se a categoria "masculino" como grupo de referência e os thetas para cada subgrupo (masculino e feminino, focal) foram equiparados em uma mesma métrica, permitindo a visualização da distribuição dos thetas $(\theta)$ no construto da ganância para homens e mulheres a partir da DGS (Figura 2). De fato, é possível identificar que os homens, quando comparados com as mulheres, se situam de forma consistente

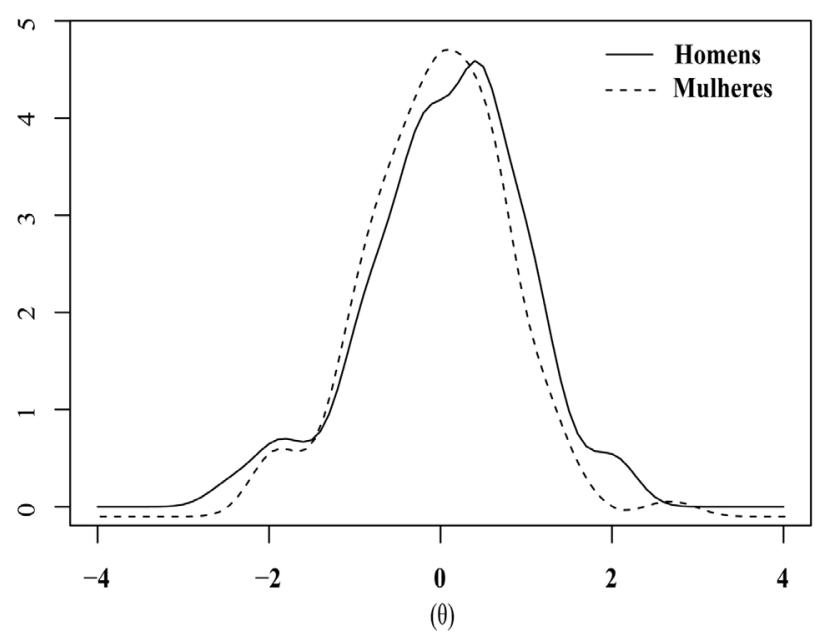

Figura 2. Distribuição dos thetas: homens e mulheres (DGS) em faixas mais elevadas do traço latente. De igual modo, níveis mais baixos do traço latente foram mais característicos do grupo do sexo feminino. Por outro lado, os resultados evidenciam ampla sobreposição nas distribuições dos thetas para estes grupos. Neste sentido, subsquentemente, procedeu-se à investigação do DIF dos itens do referido instrumento.

Efetivamente, o procedimento analítico de DIF foi realizado se estimando o modelo via simulações Monte Carlo $(\mathrm{N}=1000)$ para derivar com maior robustez estatística (e.g., controlar as taxas de ocorrência de Erro Tipo I; Choi, Gibbons, \& Crane, 2011) os dados empíricos para os critérios a priori definidos. Ressalta-se que foram necessários dois processos iterativos (método de purificação) para separar itens com suposto DIF, de modo que estes não contribuíssem para a pontuação da variável latente (theta). Na Tabela 3 estão os critérios utilizados para investigar o DIF.

Concretamente, após o processo de iteração dos dados, apenas o Item 4 ("Não importa o que já tenho, nunca estou completamente satisfeito") foi indicado como provável caso de DIF. Ao analisar os critérios adotados (Tabela 3), percebe-se que quanto ao teste de razão de verossimilhança, observou-se para o referido item uma diferença estatisticamente significativa entre os modelos 1 e 2 , bem como entre o 2 e o 3 , fato que pode apontar para a existência de DIF uniforme e não uniforme, respectivamente. Quanto às diferenças nos valores do Pseudo- $R^{2}$ e entre o parâmetro $\beta_{1}$ dos mode$\operatorname{los} 1$ e 2 , os valores em questão se situaram abaixo dos pontos de corte indicados. Assevera-se que, embora o

Tabela 3.

Métodos utilizados para detectar o funcionamento diferencial dos itens (DIF)

\begin{tabular}{ccccccccc}
\hline & \multicolumn{2}{c}{ Testes de Razão de Verossimilhança $(p)$} & & \multicolumn{3}{c}{ Diferença entre os Pseudo- $\mathrm{R}^{2}$} & \multirow{2}{*}{$\Delta\left(\beta_{1}\right)$} \\
\cline { 2 - 3 } & $\operatorname{Pr}\left(\chi_{(1 ; 2)}^{2}\right)$ & $\operatorname{Pr}\left(\chi_{(2 ; 3)}^{2}\right)$ & $\operatorname{Pr}\left(\chi_{(1 ; 3)}^{2}\right)$ & & $\mathrm{R}_{(1 ; 2)}^{2}$ & $\mathrm{R}_{(1 ; 3)}^{2}$ & $\mathrm{R}_{(2 ; 3)}^{2}$ & \\
\hline Item 1 & 0,5801 & 0,5467 & 0,3423 & & 0,0003 & 0,0013 & 0,0009 & 0,0027 \\
Item 2 & 0,3395 & 0,0833 & 0,0440 & & 0,0010 & 0,0052 & 0,0043 & 0,0050 \\
Item 3 & 0,7161 & 0,9097 & 0,8113 & & 0,0001 & 0,0002 & 0,0001 & 0,0016 \\
Item 4 & 0,0035 & 0,0118 & 0,5493 & & 0,0099 & 0,0103 & 0,0004 & 0,0134 \\
Item 5 & 0,3353 & 0,6264 & 0,9314 & & 0,0011 & 0,0011 & 0,0000 & 0,0117 \\
Item 7 & 0,3072 & 0,3527 & 0,3074 & & 0,0011 & 0,0023 & 0,0011 & 0,0101 \\
\hline
\end{tabular}

Nota. $\operatorname{Pr}\left(\chi_{(1 ; 2)}^{2}\right)$ : Comparação da razão de verossimilhança entre os passos um $(01)$ e dois $(02)$ da regressão. $\operatorname{Pr}\left(\chi_{(2 ; 3)}^{2}\right)$ Comparação da razão de verossimilhança entre os passos dois $(02)$ e três $(03)$ da regressão. $\operatorname{Pr}\left(\chi_{(1 ; 3)}^{2}\right)$ : Comparação da razão de verossimilhança entre os passos um (01) e três (03) da regressão. $\mathrm{R}_{(1 ; 2)}^{2}$ : Diferença entre os Pseudo-R2 dos passos um (01) e dois (02) da regressão. $\mathrm{R}_{(1 ; 3):}^{2}$ Diferença entre os Pseudo-R2 dos passos um (01) e três (03) da regressão. $\mathrm{R}_{(2 ; 3):}^{2}$ Diferença entre os Pseudo-R2 dos passos dois (02) e três (03) da regressão. 
Item 2 tenha apresentado diferença significativa no critério de razão de verossimilhança entre os modelos $1 \mathrm{e}$ 3 , este não foi identificado como potencial caso de DIF nos processos iterativos (método de purificação).

Em seguida, posteriormente à identificação de um item com suposto DIF, os itens da DGS foram representados graficamente por meio das Curvas Características dos itens (CCIs), conforme se observa na Figura 3. Na Figura 3-a, referente aos parâmetros de todos os itens (com e sem DIF) para homens e mulheres, observa-se um padrão análogo dos parâmetros dos itens para ambos os grupos. Em suma, tal padrão sugere impacto baixo do item que apresenta DIF quando se considera o instrumento como um todo.

Já na Figura 3-b, representando apenas os parâmetros do item com sinais de DIF no teste, o Item 4, é possível perceber o impacto nas CCIs, sugerindo a presença de DIF, inferido a partir da distância vertical entre as curvas do grupo de referência ("masculino") e focal ("feminino"). Ainda, é possível classificá-lo como um DIF do tipo uniforme, tendo em vista que as CCIs não se cruzam em qualquer ponto do intervalo do traço latente, indicando haver diferenças para o parâmetro b (dificuldade), mas não em termos de discriminação (parâmetro a). Assim, identifica-se maior probabilidade de endosso deste item, por parte dos homens, em uma larga faixa do continuum do traço latente. Observa-se apenas um nível de probabilidade de concordância similar, para ambos os grupos, em níveis mais elevados do traço mensurado.

\section{Discussão}

O presente estudo objetivou conhecer evidências de validade com base na estrutura interna e precisão da Dispositional Greed Scale (DGS; Seuntjens et al., 2015), assim como investigar os parâmetros de seus itens no contexto brasileiro. Esta proposta foi motivada pela inexistência de estudos empíricos acerca do construto da ganância no Brasil. Neste sentido, os esforços se concentraram inicialmente na adaptação e validação de uma medida com propriedades psicométricas (e.g., estrutura interna e parâmetros de itens) adequadas para mensurar o referido construto. Os achados principais são a seguir tratados.

\section{Evidências da Estrutura Interna e Fidedignidade da DGS}

Inicialmente, verificou-se uma estrutura unifatorial da DGS, sendo coerente teórica e empiricamente com estudos conduzidos com essa finalidade (Krekel \& Pandelaere, 2015; Seuntjens et al., 2015). Nesta ocasião, empregou-se o método Hull (Ceulemans \& Kiers, 2006) para determinar tal estrutura em razão de sua maior eficácia demonstrada em estudos de simulação frente aos procedimentos recorrentes na literatura para retenção de número de fatores (e.g., eigenvalue $>1$, scree plot,
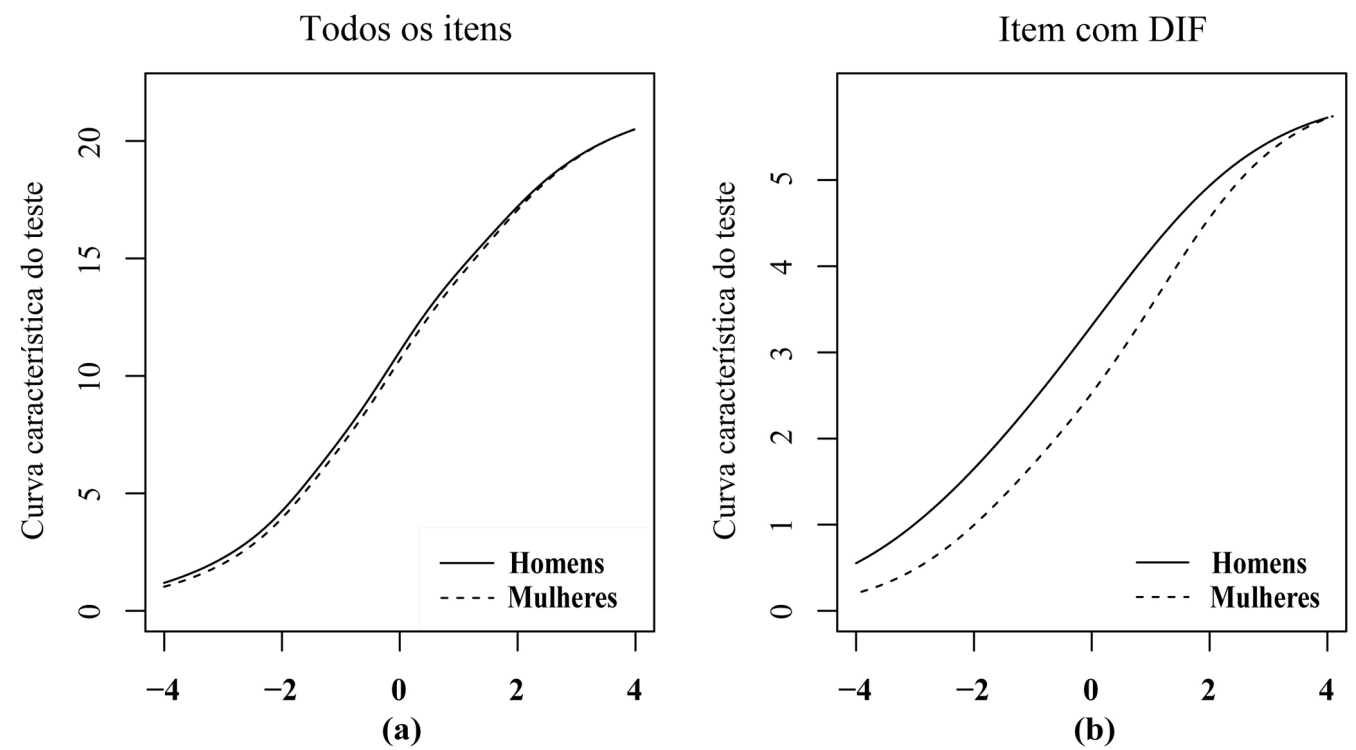

Figura 3. Impacto dos itens com suposto DIF nas curvas características do teste 
parallel analysis e minimum average partial; Lorenzo-Seva et al., 2011). Ressalta-se que o item 6 não apresentou saturação mínima adequada. Em relação à sua redação, trata-se do único item que faz referência a um elemento específico, no caso o dinheiro. Desta forma, na amostra brasileira este item pode ter sido concebido como algo que remeta unicamente a bens materiais, isto é, aludindo ao construto do materialismo (Richins, 2004) e não à ganância, uma vez que esta última não se restringe a elementos materiais (Seuntjens et al., 2015; Tickle, 2004).

Quanto à consistência interna, considerando a matriz de correlações policóricas, observou-se um índice satisfatório do ômega de McDonald (Cohen, Swerdlik, \& Sturman, 2014). Assevera-se que este indicador apresenta uma estimativa de maior acurácia da confiabilidade de uma medida em contraposição ao alfa de Cronbach que, em certas condições, pode subestimar a consistência interna de instrumentos (Sijtsma, 2009). Portanto, o índice de ômega de McDonald consiste em uma alternativa prática de precisão de instrumentos psicológicos (Dunn, Baguley, Brunsden, 2014), permitindo maior margem de segurança na investigação deste parâmetro de medidas psicológicas.

\section{Parâmetros e Funcionamento Diferencial dos Itens da DGS}

Inicialmente, em relação aos procedimentos estatísticos ancorados na TRI, calculou-se o índice de discriminação dos itens da DGS. A partir da classificação proposta por Baker (2001), o Item 2 apresentou um valor correspondente a uma discriminação muito alta, ao passo que o Item 1 apresentou alta discriminação. Os demais itens (itens 3, 4, 5 e 7) foram classificados com apesentando discriminação moderada. Por outro lado, em sua totalidade os itens da DGS apresentaram alta discriminação, atestando a qualidade métrica da medida na diferenciação de pessoas com magnitudes próximas no traço latente da ganância.

Quanto ao parâmetro de dificuldade, a partir das orientações de Pasquali (2007), os itens 2, 3 e 5 apresentaram menor índice de dificuldade, isto é, apresentaram maiores probabilidades de serem endossados pelos indivíduos. Tais resultados podem indicar que tal conjunto de itens associa a ganância com uma tendência geral de autodesenvolvimento; de fato, alguns autores argumentam que ser ganancioso é vital para o bem-estar humano, relacionando-se com questões socialmente valorizadas em geral (e.g., crescimento econômico; Greenfeld, 2001). Já os itens 1, 4 e 7 foram considerados os mais difíceis. Dentre eles, no que tange à semântica, destaca-se o item 7 que faz menção direta à palavra "ganância". No estudo de Seuntjens et al. (2015), a DGS se correlacionou negativamente com a medida de desejabilidade social, indicando ser esta uma qualidade socialmente reprovável. Um fato que pode explicar a menor aceitação de tais itens se relaciona com a conotação negativa que tal termo possui. A ganância é avaliada negativamente nas principais tradições religiosas (Seuntjens et al., 2014), sendo entendida, de igual modo, como a causa de alguns pecados (e.g., raiva, inveja, gula, luxúria, orgulho; Tickle, 2004). Ainda, os itens da DGS exigiram baixo/moderado traço latente, garantindo a aplicabilidade da medida para mensurar a ganância em amplo espectro populacional no contexto brasileiro.

No que se refere à análise de DIF, apenas o critério da razão de verossimilhança indicou a presença de funcionamento diferencial, mais especificamente no item 4 da DGS. A análise das CCIs deste item indicou um DIF do tipo uniforme. Em síntese, os homens apresentaram probabilidade mais acentuada de endossar tal item do que as mulheres no continum do traço latente, havendo apenas um padrão de endosso similar para pessoas com níveis mais elevados de ganância. Tal cenário é congruente com o fato de que os homens tendem a demonstrar maiores níveis de ganância (Krekels \& Pandelaere, 2015), atestando o viés da variável sexo no construto.

A menor propensão de concordância das mulheres frente ao item com DIF pode ser explicado por diversos fatores. Por exemplo, alguns estudos (e.g., Cadsby \& Maynes, 1998; Seguino, Stevens, \& Lutz, 1996) já verificaram que as mulheres possuem uma visão menos positiva de tal construto, relatando maior desconforto psicológico do que os homens após o endosso ou o envolvimento em situações que correspondam à ganância. Outra possível explicação se refere ao fato de que o construto da ganância implica em processos de regulação de cooperação social (e.g., conflitos entre interesses individuais e coletivos; Simpson, 2003). Assim, as mulheres apresentam maiores níveis de empatia, cooperação e menos comportamentos autocentrados do que os homens (Seguino et al., 1996), expressando, portanto, menores níveis de ganância.

Em suma, embora o Item 4 tenha se apresentado enquanto um adequado indicador do construto da ganância, este se mostrou, em termos de magnitude de traço latente, mais severo/difícil para o grupo do sexo feminino. Embora tal item não tenha apresentando um alto impacto de DIF no instrumento em geral, adverte-se para a necessidade de sua calibração individual

Psico-USF, Bragança Paulista, v. 24, n. 3, p. 489-500, jul./set. 2019 
(controlando o efeito de viés), bem como a revisão de seu conteúdo. Por outro lado, para os demais itens da DGS, assegurou-se igual probabilidade de endosso para homens e mulheres, evitando vieses de resposta quanto ao sexo dos participantes, indicando que eventuais diferenças no construto resultam de níveis do traço latente das pessoas e não pelo fato de pertencerem a dado subgrupo. Estas recomendações resguardarão a interpretação de resultados em estudos futuros que tenham em conta as diferenças entre sexo frente ao construto da ganância no contexto brasileiro (Martinková et al., 2017).

\section{Considerações finais e direcionamentos futuros}

Finalmente, quanto às limitações do presente estudo, menciona-se, por exemplo, a não representatividade das amostras, impossibilitando generalizações das conclusões ora apresentadas. Por outro lado, confia-se que os objetivos tenham sido alcançados, fornecendo-se uma escala psicometricamente satisfatória para mensurar a ganância no Brasil, sendo a interpretação de seus escores, no geral, equivalente quanto ao sexo dos participantes. Como direcionamentos futuros, sugerem-se estudos que investiguem evidências complementares de validades convergente e discriminante da DGS, o DIF diante em outras variáveis sociodemográficas (e.g., faixa etária, orientação política, etc.) bem como considerem medidas implícitas, buscando reduzir o efeito do componente da desejabilidade social da ganância. Ademais, tendo em vista a natureza sociopsicológica deste construto, recomenda-se conhecer em que medida este pode estar relacionado com variáveis de natureza mais social, como os valores humanos. Nesta direção, espera-se que maiores níveis de ganância predisponham maior endosso de uma orientação valorativa guiada por metas pessoais (e.g., êxito, poder e prestígio; Gouveia, 2013).

\section{Referências}

Baker, F. B. (2001). The basics of item response theory (2nd ed.). Washington, DC: Eric Clearinghouse on Assessment and Evaluation.

Borsa, J. C., Damásio, B. F., \& Bandeira, D. R. (2012). Adaptação e validação de instrumentos psicológicos entre culturas: algumas considerações. Paidéia, 22(53), 423-432. doi: 10.1590/S0103-863X2012000300014

Psico-USF, Bragança Paulista, v. 24, n. 3, p. 489-500, jul./set. 2019
Cadsby, B., \& Maynes, E. (1998). Gender and free riding in a threshold public goods game: Experimental evidence. Journal of Economic Behavior \& Organization, 34(4), 603-640. doi: 10.1016/ S0167-2681(97)00010-3

Ceulemans, E., \& Kiers, H. A. L. (2006). Selecting among three-mode principal component models of different types and complexities: A numerical convex hull based method. British Journal of Mathematical and Statistical Psychology, 59, 133-150. doi: 10.1348/000711005X64817

Chalmers, R. P. (2012). Mirt: A Multidimensional Item Response Theory Package for the R Environment. Journal of Statistical Software, 48(6), 1-29. doi: 10.18637/jss.v048.i06

Choi, S. W., Gibbons, L. E., \& Crane, P. K. (2011). Lordif: An R package for detecting differential item functioning using iterative hybrid ordinal logistic regression/item response theory and Monte Carlo simulations. Journal of statistical software, 39(8), 1-30. Recuperado de: https://www.ncbi.nlm.nih.gov/ pubmed/21572908

Choi, S. W., Gibbons, L. E. \& Crane, P. K. (2016). Lordif: Logistic Ordinal Regression Differential Item Functioning using IRT. Recuperado de: ftp://cran.r-project.org/pub/R/web/packages/lordif/lordif. pdf

Cohen, R. J., Swerdlik, M. E., \& Sturman, E. D. (2014). Testagem e Avaliação Psicológica: Introdução a Testes e Medidas. (8. ed) São Paulo: AMGH.

Crane, P. K., van Belle, G., \& Larson, E. B. (2004). Test bias in a cognitive test: Differential item functioning in the CASI. Statistics in Medicine, 23(2), 241-256. doi: 10.1002/sim.1713

Crane, P. K., Hart, D. L., Gibbons, L. E., \& Cook, K. F. (2006). A 37-item shoulder functional status item pool had negligible differential item functioning. Journal of Clinical Epidemiology, 59(5), 478-484. doi: 10.1016/j.jclinepi.2005.10.007

Crocker, J., Canevello, A., \& Brown, A. A. (2017). Social motivation: Costs and benefits of selfishness and otherishness. Annual review of psychology, 68, 299325. doi: 10.1146/annurev-psych-010416-044145.

Dunn, T. J., Baguley, T., Brunsden, V. (2014). From alpha to omega: A practical solution to the pervasive problem of internal consistency estimation. British 
Journal of Psychology, 105, 399-412. doi: 10.1111/ bjop.12046

Eckel, C. C., \& Grossman, P. J. (1998). Are women less selfish than men? Evidence from dictator experiments. The economic journal, 108(448), 726-735. doi: 10.1111/1468-0297.00311

Gouveia, V. V. (2013). Teoria funcionalista dos valores bumanos: Fundamentos, aplicações e perspectivas. São Paulo: Casa do Psicólogo.

Greenfeld, L. (2001). The spirit of capitalism: Nationalism and economic growth. Cambridge, MA: Harvard University Press.

Haynes, K. T., Josefy, M., \& Hitt, M. A. (2015). Tipping Point: Managers' Self-Interest, Greed, and Altruism. Journal of Leadership \& Organizational Studies, 22(3), 265-279. doi: 10.1177/1548051815585171

Holgado-Tello, F. P., Chacón-Moscoso, S., Barbero-García, I., \& Vila-Abad, E. (2010). Polychoric versus Pearson correlations in exploratory and confirmatory factor analysis of ordinal variables. Quality \& Quantity, 44(1), 153-166. doi: 10.1007/s11135-008-9190-y

International Test Commission. (2010). Guidelines for Translating and Adapting Tests. Recuperado de: http://www.intestcom.org

Jodoin, M. G., \& Gierl, M. J. (2001). Evaluating Type I Error and Power Rates Using an Effect Size Measure With the Logistic Regression Procedure for DIF Detection. Applied Measurement in Education, 14(4), 329-349. doi: 10.1207/S15324818AME1404_2

Krekels, G., \& Pandelaere, M. (2015). Dispositional greed. Personality and Individual Differences, 74, 225230, 2005. doi: 10.1016/j.paid.2014.10.036

Lorenzo-Seva, U., \& Ferrando, P. J. (2013). FACTOR 9.2: A Comprehensive Program for Fitting Exploratory and Semiconfirmatory Factor Analysis and IRT Models. Applied Psychological Measurement, 37(6), 497-498. doi:10.1177/0146621613487794

Lorenzo-Seva, U., Timmerman, M. E., \& Kiers, H. A. L. (2011). The Hull Method for Selecting the Number of Common Factors. Multivariate Behavioral Research, 46(2), 340-364. doi: 10.1080/00273171.2011.564527

Martinková, P., Drabinová, A., Liaw, Y. L., Sanders, E. A., McFarland, J. L., \& Price, R. M. (2017).
Checking equity: Why differential item functioning analysis should be a routine part of developing conceptual assessments. CBE-Life Sciences Education, 16(2), 1-13. doi: 10.1187/cbe.16-10-0307.

Marcus, D. K., \& Zeighler-Hill, V. (2015). A Big Tent of Dark Personality Traits. Social and Personality Psychology Compass, 9(8), 434-446. doi: 10.1111/ spc3.12185

Mussel, P. \& Hewig, J. (2016). The life and times of individuals scoring high and low on dispositional greed. Journal of Research in Personality, 64, 52-60. doi: 10.1016/j.jrp.2016.07.002

Pasquali, L. (2007). Teoria de resposta ao item: teoria, procedimentos e aplicações. Brasília: LabPAM/Unb.

R Development Core Team. (2015). R: A language and environment for statistical computing. Recuperado de: https://cran.r-project.org/doc/manuals/fullrefman.pdf

Reavis, C. (2012). The Global Financial Crisis of 2008: The Role of Greed, Fear, and Oligarchs. MIT Sloan Management Review, 16, 1-22. Recuperado de: https:/ / mitsloan.mit.edu/LearningEdge/CaseDocs $/ 09-093 \% 20$ The $\% 20$ Financial $\% 20$ Crisis $\% 20$ of $\% 202008$.rev.pdf

Richins, M. L. (2004). The material values scale: Measurement properties and development of a short form. Journal of Consumer Research, 31(1), 209 -219. doi: 10.1086/383436

Samejima, F. (1969). Estimation of latent ability using a response pattern of graded scores. Psychometrika Monograph Supplement, 34(4), 100-114. doi: 10.1002/ j.2333-8504.1968.tb00153.x

Seguino, S., Stevens, T., \& Lutz, M. (1996). Gender and cooperative behavior: Economic man rides alone. Feminist Economics, 2(1), 1-21. doi: $10.1080 / 738552683$

Seuntjens, T. G., Zeelenberg, M. Breugelmans, S. M., \& van de Ven, N. (2014). Defining Greed. British Journal of Psychology, 106(3), 505-525. doi: 10.1111/ bjop.12100

Seuntjens, T. G., Zeelenberg, M., van de Ven, N., \& Breugelmans, S. M. (2015). Dispositional Greed. Journal of Personality and Social Psychology, 108(6), 917-933. doi: 10.1037/pspp0000031 
Seuntjens, T. G., Van de Ven, N., Zeelenber, M., \& van der Schors, A. (2016). Greed and adolescent financial behavior. Journal of Economic Psychology, 57, 1-2. doi: 10.1016/j.joep.2016.09.002

Sijtsma, K. (2009). On the use, the misuse, and the very limited usefulness of Cronbach's alpha. Psychometrika, 74, 107-120. doi: 10.1007/s11336-008-9101-0

Simpson, B. (2003). Sex, fear, and greed: A social dilemma analysis of gender and cooperation. Social forces, 82(1), 35-52. Recuperado de: https://www. jstor.org/stable/3598137

Swaminathan, H., \& Rogers H. J. (1990). Detecting Differential Item Functioning using logistic regression procedures. Journal of Educational Measurement,
27(4), 361-370. doi: 10.1111/j.1745-3984.1990. tb00754.x

Tickle, P. (2004). Greed: The seven deadly sins. Oxford, United Kingdom: Oxford University Press.

Walker, C. M. (2011). What's the DIF? Why differential item functioning analyses are an important part of instrument development and validation. Journal of Psychoeducational Assesment, 29(4), 364-376. doi: 10.1177/0734282911406666

Weiszflög, W. (2015). Michaelis: Dicionário Brasileiro da Língua Portuguesa. São Paulo: Editora Melhoramentos Ltda.

Recebido em: 22/12/2017

Reformulado em: 16/08/2018

Aprovado em: 28/09/2018 
Sobre os autores:

Leogildo Alves Freires é doutor em Psicologia Social pela Universidade Federal da Paraíba (UFPB) e professor classe A, Nível II (Adjunto A) do Instituto de Psicologia da Universidade Federal de Alagoas (UFAL). Atualmente coordena o Laboratório de Psicometria e Avaliação Psicológica (LAPAP/UFAL).

ORCID iD 0000-0001-5149-2648

E-mail: leogildo.freires@ip.ufal.br

Gleidson Diego Lopes Loureto possui graduação em Psicologia (2016) pela Universidade Federal de Roraima (UFRR). Atualmente cursa mestrado em Psicologia Social na Universidade Federal da Paraíba (UFPB) sob orientação do Prof. Dr. Valdiney Veloso Gouveia. É colaborador do núcleo de Pesquisa Bases Normativas do Comportamento Social (BNCS). Seus interesses de pesquisa se centram nas áreas de Avaliação Psicológica, Psicologia Social e Análise de dados.

ORCID iD 0000-0002-0889-6097

E-mail: diegoloureto.dl@gmail.com

Maria Gabriela Costa Ribeiro possui graduação em Psicologia (2016) pela Universidade Federal da Paraíba (UFPB). Atualmente faz mestrado no Programa de pós-graduação em Psicologia Social da mesma instituição e é integrante do núcleo de pesquisas Bases Normativas do Comportamento Social (BNCS), coordenado pelo professor Dr. Valdiney Veloso Gouveia. Alguns interesses em pesquisas são: Avaliação Psicológica, Psicologia Social e Psicologia Positiva. ORCID iD 0000-0001-6920-9070

E-mail: mariagabicr@gmail.com

Layrtthon Carlos de Oliveira Santos é doutor em Psicologia Social pela Univerdidade Federal da Paraíba (UFPB) e colaborador do núcleo de pesquisa Bases Normativas do Comportamento Social (BNCS). Atualmente é professor substituto no Centro de Ciências Exatas e Sociais Aplicadas (CCEA) da Universidade Estadual da Paraíba (UEPB). Seus interesses de pesquisa têm relacionado a Teoria Funcionalista dos Valores Humanos a diferentes fenômenos como intenção de voto, religiosidade e alimentação.

ORCID iD 0000-0002-9061-4879

E-mail: layrtthon.oliveira@gmail.com

Valdiney Veloso Gouveia é doutor em Psicologia Social pela Universidade Complutense de Madrid (1998), professor titular do Departamento de Psicologia da Universidade Federal da Paraíba e bolsista de produtividade (1A) do CNPq. Seus interesses de pesquisa se centram nas áreas de Psicologia Social (estruturas sociais; indivíduos) e Avaliação Psicológica (construção e adaptação de escalas e testes).

ORCID iD 0000-0003-2107-5848

E-mail:vvgouveia@gmail.com

Contato com os autores:

Prof. Dr. Leogildo Alves Freires

Universidade Federal de Alagoas - Campus AC Simões

Instituto de Psicologia, Sala 5

Av. Lourival Melo Mota, S/N

CEP: 57072-970 